\title{
Retinal microangiopathy in pigmented paravenous chorioretinal atrophy
}

\author{
SURESH R LIMAYE AND MUNEERA A MAHMOOD \\ From the Ophthalmology Service, DC General Hospital and Center for Sight, Georgetown University Medical \\ Center, Washington, DC, USA
}

SUMMARY This report describes an atypical case of pigmented paravenous chorioretinal atrophy, associated with focal progressive peripheral retinal microangiopathy, in a 51-year-old black female. The eyes were asymmetrically involved. Although several cases have been reported with typical features of this uncommon entity, none of them have been known to be associated with retinal microangiopathy. The occurrence of such microangiopathy supports the hypothesis that damage to the retinal photoreceptors may induce retinal microangiopathy; as suggested in other clinical and experimental studies.

Pigmented paravenous chorioretinal atrophy is an asymptomatic disease characterised by the presence of bilaterally symmetrical chorioretinal atrophy along the distribution of the retinal veins with variable pigment migration. The disease progresses slowly and its aetiology remains unknown. As the condition is restricted to the paravenous area, it is thought not to be a sight-threatening generalised degenerative disorder of the retina.' Between 1973 and 1980 we examined three patients with pigmented paravenous chorioretinal atrophy, only one of whom had an unusual progressive, peripheral, retinal microangiopathy in the more involved eye. Such a case of retinal microangiopathy has not to our knowledge been previously reported.

\section{Case report}

A 51-year-old black female from the West Indies was examined for a routine eye examination in September 1976. She denied symptoms of night blindness or previous ocular disease. Her past medical history was also unremarkable, and there was no family history of night blindness or any other ocular diseases. Visual acuity was $6 / 6$ in each eye. External examination did not reveal any abnormality. Extraocular motility was full, and she was orthophoric for distance and near. Slit-lamp exami-

Correspondence to Suresh R Limaye, MD, District of Columbia General Hospital, Ophthalmology Service, Room 3325, Core Building, 19th Street and Massachusetts Avenue, SE, Washington, DC 20003, USA. nation showed only minor cuneiform cataracts in each eye. Intraocular pressure by applanation tonometry was $16 \mathrm{mmHg}$ in each eye.

The fundus of the right eye showed typical paravenous corpuscular pigmentation, with chorioretinal atrophy extending from the optic disc to the equator and beyond (Fig. 1). In the temporal periphery microaneurysms and telangiectasia of the retinal vessel was seen, but without exudates (Fig. 2).

The fundus of the left eye, however, showed only one isolated patch of chorioretinal atrophy along the inferior temporal vein (Fig. 3).

The visual field of the right eye was markedly constricted, especially on the nasal aspect, and that of the left eye was full on Goldmann perimetry (Figs. $4 a, 4 b$ ). A rod dominant (scotopic) electroretinogram of the right eye revealed subnormal b-wave amplitude $(150 \mu \mathrm{v})$ with a normal implicit time (45 $\mathrm{m} / \mathrm{s})$. The electroretinogram of the left eye in a rod dominated condition showed a b-wave amplitude of $350 \mu \mathrm{v}$ with an implicit time of $45 \mathrm{~m} / \mathrm{s}$. Electrooculography showed the light peak to dark trough ratio of 2.0 for the right eye and 2.3 for the left eye.

Fluorescein angiography of the right eye showed an extensive area of choriocapillaris atrophy with prominantly visible choroidal vessels along the major retinal veins adjacent to the disc in an early arterial phase. In the arteriovenous phase hyperfluorescence was seen at the edge of the atrophic area and hypofluorescence was seen corresponding to the areas of pigment migration (Fig. 5).

Systemic examination was unremarkable and 
Fig. 1 Right fundus: a typical paravenous chorioretinal atrophy with pigment migration, and uninvolved macular area.



routine laboratory tests, including serology for syphilis and skin test for tuberculosis, were negative. Haemoglobin electrophoresis did not reveal any abnormal haemoglobinopathy.

Three years after the initial examination she was found to have intraretinal hard exudates in the area of previously noted microaneurysms and telangiectasia, namely, in the temporal periphery of the fundus (Fig. 6). Fluorescein angiography of the peripheral fundus showed areas of capillary nonperfusion, arteriovenous communications, and microaneurysms (Fig. 7).

\section{Discussion}

Pigmented paravenous chorioretinal atrophy is an uncommon disease of unknown aetiology. The first case of this disorder was described by Brown in 1937 under the name of retinochoroiditis radiata. ${ }^{2}$ In 1962 Franceschetti coined the term pigmented paravenous choroiditis. ${ }^{3}$ Several other cases have been published some under different names. ${ }^{46}$ Noble and Carr recently reported six additional cases and reaffirmed its characteristics, ${ }^{1}$ previously well described by Krill.' The linear extent of chorioretinal atrophy and paravenous pigmentation is remarkably variable among these cases. A slow progression of the disease without involvement of the macular area has been emphasised. ${ }^{5}$

This case report, however, is unusual because such an asymmetrical involvement is rare, and the presence of retinal microangiopathy has not been described before. As in previously reported cases, there was no evidence of intraocular inflammation, and the laboratory tests for associated systemic disease were negative. The patient also denied 


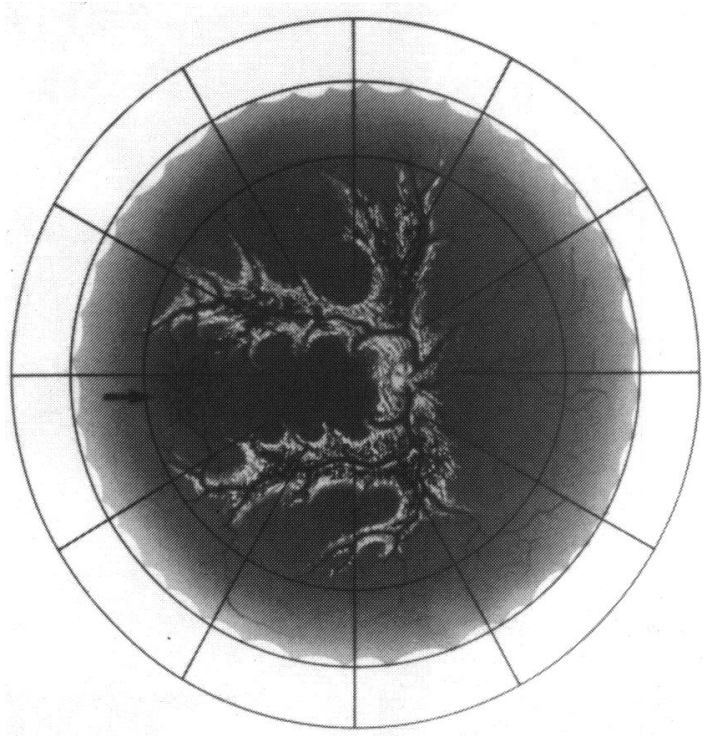

Fig. 2 Microaneurysm and telangiectasia seen in the temporal periphery (arrow) of the right eye.

having a family history of any ocular disorder. At the initial examination only telangiectatic microvascular changes were observed, without any intraretinal exudate. Three years later a collection of hard exudates in the inner retina was observed. Fluorescein angiography in this area showed capillary non-perfusion, arteriovenous communication, microaneurysmal dilatation and vessel wall staining. A microangiopathy associated with other disorders such as diabetes mellitus, vein occlusion, sickle cell retinopathy, sarcoidosis, Eales' disease, and radiation retinopathy was excluded on the basis of clinical examination and laboratory investigation. Unfortunately not all her family members were available for eye examination, though they claimed to have 'good eyes'.

It is noteworthy that the retinal microangiopathy, though not previously described with pigmented paravenous chorioretinal atrophy, has been noted by Cleary et al. ${ }^{8}$ in five cases of congenital hypertrophy of retinal pigment epithelium. Similar retinal vascular abnormalities have been described in rats with hereditary visual cell degeneration, ${ }^{9}$ the light damaged rat retina, ${ }^{10}$ and after argon and ruby laser photocoagulation of monkey retina. ${ }^{.1}$ Dantzker and Gerstein " observed obliteration of retinal capillaries in cats with iodoacetate induced photoreceptor damage. Telangiectatic changes are known to occur on the optic nerve head in patients with cone-rod degeneration. ${ }^{12}$ Therefore it is postulated that in our patient the presence of microangiopathy may signify photoreceptor damage. Whether photoreceptor damage was secondary to primary degeneration of the retinal pigment epithelium is debatable. A marked constriction of the visual field and a subnormal ERG are, however, indicative of photoreceptor damage. It is not clear why the disease has appeared so asymmetrically. The pathogenesis of pigmented paravenous chorioretinal atrophy by itself and in association with retinal microangiopathy remains unresolved.

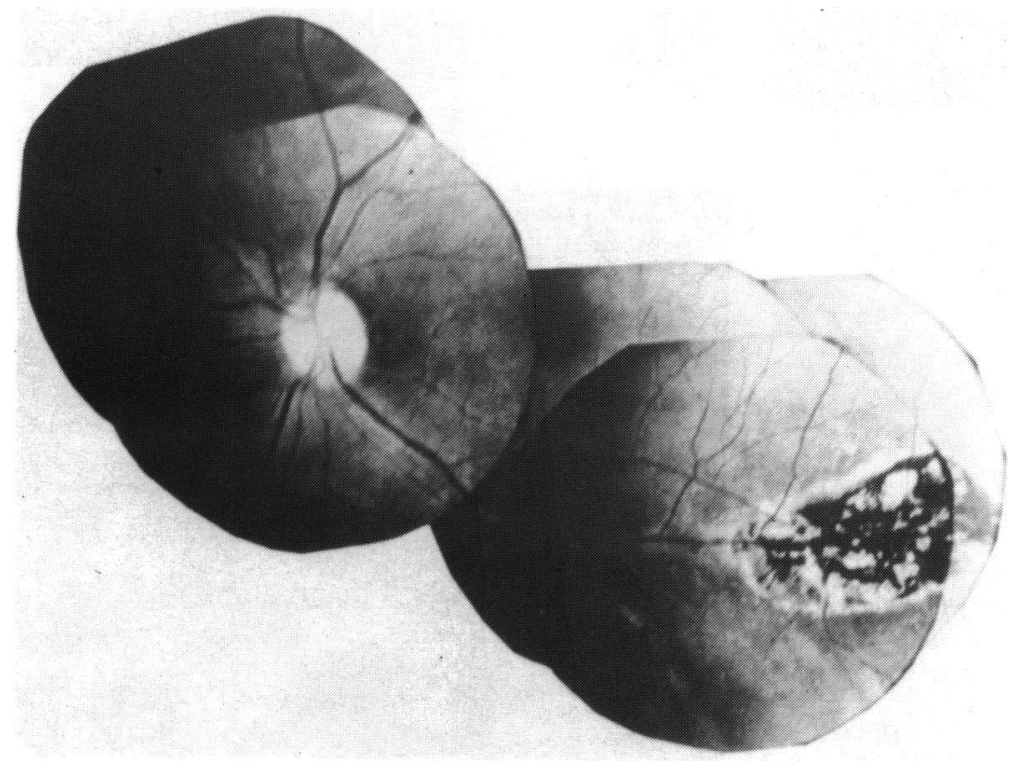

Fig. 3 Left fundus: only an isolated patch of chorioretinal atrophy seen. 


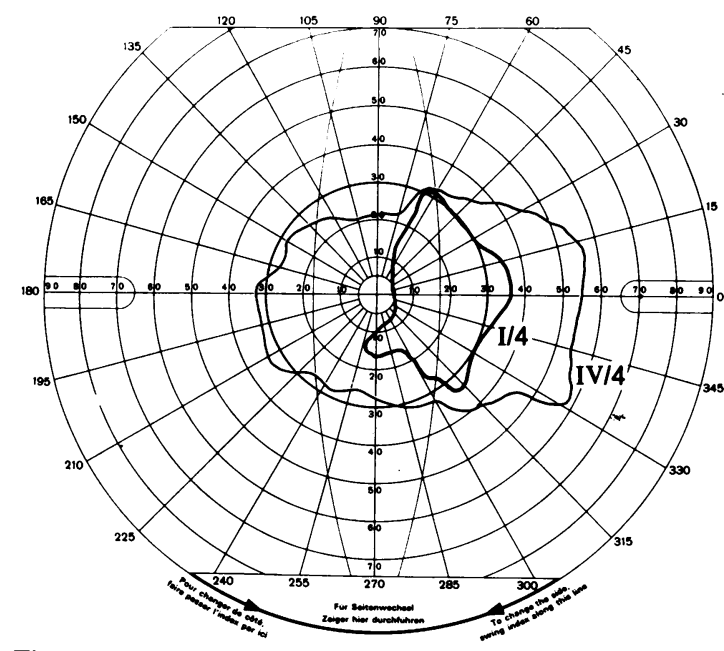

Fig. 4a

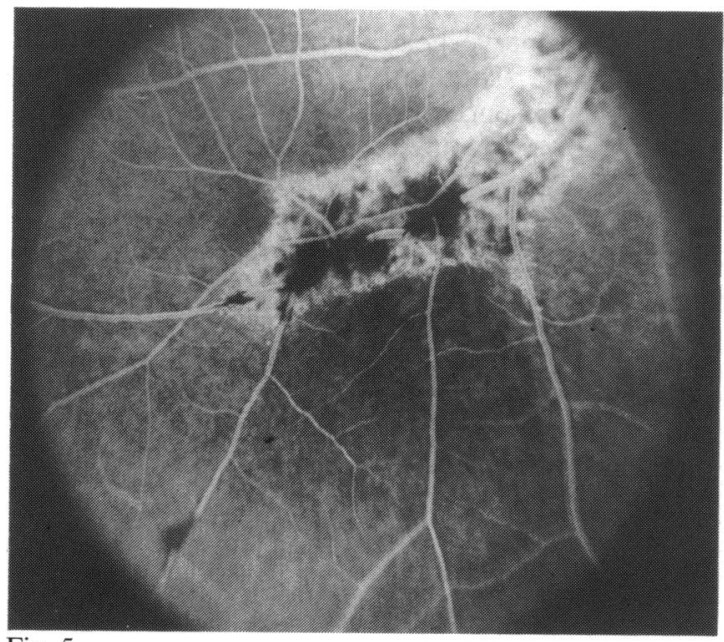

Fig. 5

Fig. 4A Visual field of the right eye.

Fig. 4B Visual field of the left eye.

Fig. 5 Fluorescein angiogram of the right eye showing transmitted hyperfluorescence and blocked fluorescence.

Fig. 6 Temporal periphery of the right eye showing accumulation of hard exudates not seen on the initial examination.

Fig. 7 Fluorescein angiogram of the temporal periphery showing capillary non-perfusion, microaneurysm, and arteriovenous communications.

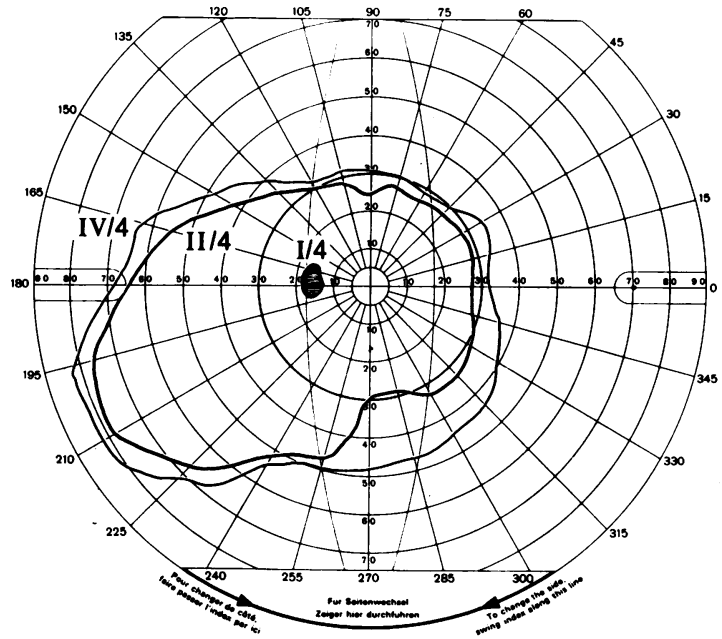

Fig. 4b

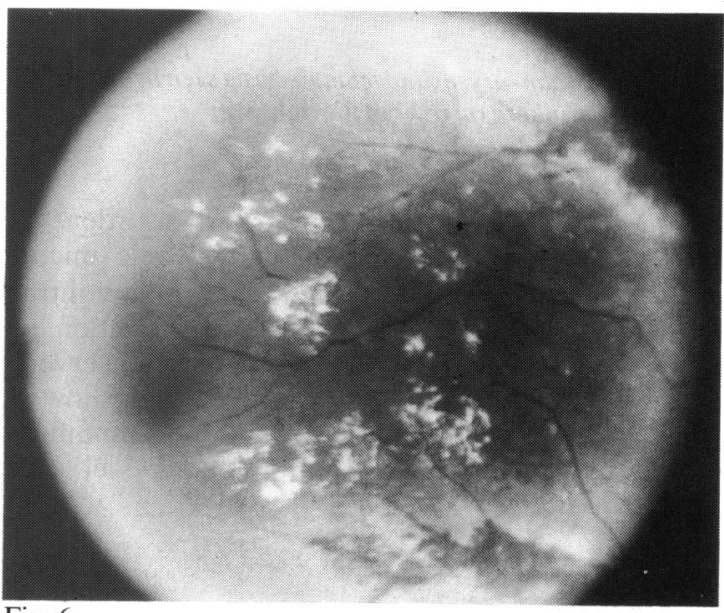

Fig. 6

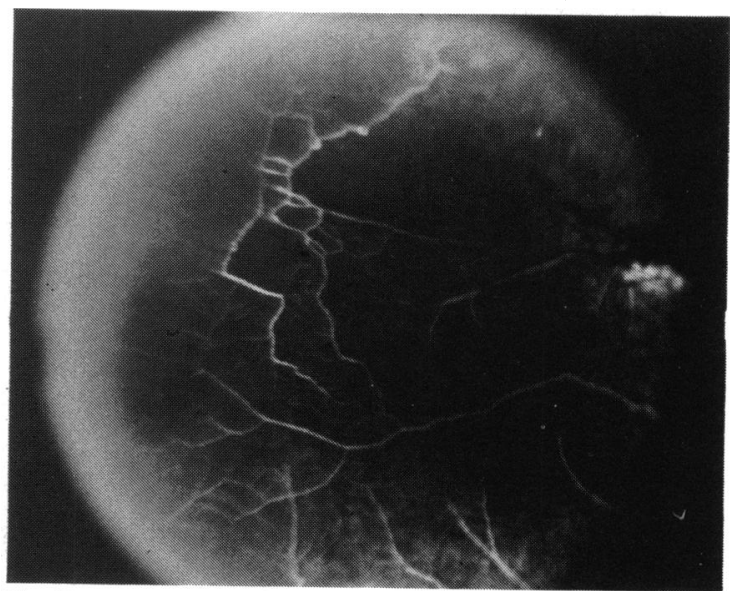

Fig. 7 


\section{References}

1 Noble KG, Carr RE. Pigmented paravenous chorioretinal atrophy. Am J Ophthalmol 1983; 96: 338-44.

2 Brown TH. Retino-choroiditis radiata. Br J Ophthalmol 1937; 21: $645-8$.

3 Franceschetti A. A curious affection of the fundus oculi Helicoid peripapillary chorioretinal degeneration. Its relation to pigmentary paravenous chorioretinal degenerations. Doc Ophthalmol 1962; 16: 81-110.

4 Morgan OG. Congenital pigmentation of the retina. Proc $R$ Soc Med 1948; 41: 726-7.

5 Pearlman JT, Kamin DF, Kopelow SM. Pigmented paravenous retinochoroidal atrophy. Am J Ophthalmol 1975; 80: 630-5.

6 Skalka HW. Hereditary pigmented paravenous chorioretinal atrophy. Am J Ophthalmol 1979; 87: 286-91.

7 Krill AE. Krill's hereditary retinal and choroidal diseases. Hagerstown: Harper and Row, 1977; 2: 625-35.
8 Cleary PE, Gregor Z, Bird AC. Retinal vascular changes in congenital hypertrophy of retinal pigment epithelium. $\mathrm{Br} J$ Ophthalmol 1976; 60: 499-503.

9 Gerstein DD, Dantzker DR. Retinal vascular changes in hereditary visual cell degeneration in dystrophic rats. Arch Ophthalmol 1969; 81: 99-105.

10 Dantzker DR, Gerstein DD. Retinal vascular changes following toxic effects on visual cells and pigment epithelium. Arch Ophthalmol 1969; 81: 106-14.

11 Okisaka S, Kuwabara T, Aiello LM. The effects of laser photocoagulation in the retinal capillaries. Am J Ophthalmol 1975; 80: 591-601.

12 Heckenlively, JR, Martin DA, Rosales TO. Telangiectasia and optic atrophy in cone-rod degeneration. Arch Ophthalmol 1981; 99: 1983-91.

Accepted for publication 9 October 1986. 